\title{
Correction to: Microbiome and nitrate removal processes by microorganisms on the ancient Preah Vihear temple of Cambodia revealed by metagenomics and $\mathrm{N}-15$ isotope analyses
}

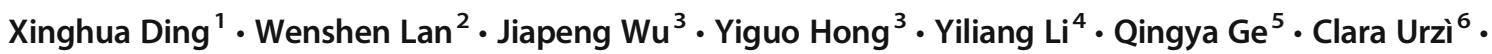 \\ Yoko Katayama ${ }^{7} \cdot$ Ji-Dong Gu ${ }^{8}$ (D)
}

Published online: 17 October 2020

(C) Springer-Verlag GmbH Germany, part of Springer Nature 2020

\section{Correction to: Applied Microbiology and Biotechnology https://doi.org/10.1007/s00253-020-10886-4}

The published online version contains mistake for the authors correction to change the name from Clara Uriz to Clara Urzì was missed.

The original publication was revised.

Publisher's note Springer Nature remains neutral with regard to jurisdictional claims in published maps and institutional affiliations.

The original article can be found online at https://doi.org/10.1007/ s00253-020-10886-4

Ji-Dong Gu

jidong.gu@gtiit.edu.cn

1 Laboratory of Environmental Microbiology and Toxicology, School of Biological Sciences, The University of Hong Kong, SAR, Pokfulam Road, Hong Kong, People's Republic of China

2 Shenzhen R\&D Key Laboratory of Alien Pest Detection Technology, The Shenzhen Academy of Inspection and Quarantine, Food Inspection and Quarantine Center of Shenzhen Customs, 1011 Fuqiang Road, Shenzhen 518045, People's Republic of China

3 Institute of Environmental Research At Greater Bay Area, Key Laboratory for Water Quality and Conservation of the Pearl River Delta, Ministry of Education, Guangzhou University, Guangzhou 510006, People's Republic of China
Department of Earth Sciences, The University of Hong Kong, SAR, Pokfulam Road, Hong Kong, People's Republic of China

5 ChineseAcademy of Cultural Heritage, 2 Gaoyuan Street, Chaoyang District, Beijing 100029, People's Republic of China

6 Department of Chemical, Biological, Pharmaceutical and Environmental Sciences, University of Messina, Viale F. Stagno d'Alcontres 31, 98166 Messina, Italy

7 Tokyo National Research Institute for Cultural Properties, 13-43 Ueno Park, Taito-ku, Tokyo 110-8713, Japan

8 Environmental Engineering, Guangdong Technion Israel Institute of Technology, 241 Daxue Road, Shantou 515063, Guangdong, People's Republic of China 